\title{
Mutual diffusion in concentrated liquid solutions: A new model based on cluster theory
}

\author{
Amin Kamgar ${ }^{1}$, Ali Bakhtyari ${ }^{1}$, Sajjad Mohsenpour ${ }^{1}$, Carmine D'Agostino², Geoff Moggridge ${ }^{2}$, Mohammad \\ Reza Rahimpour $^{1 *}$ \\ ${ }^{I}$ Chemical Engineering Department, Shiraz University, Shiraz 71345, Iran \\ ${ }^{2}$ Department of Chemical Engineering and Biotechnology, University of Cambridge, Pembroke Street, \\ Cambridge CB2 3RA, UK
}

\section{Abstract}

In dilute solutions, diffusion is dominated by motion of single molecules. Conversely, in nonideal concentrated solutions, mass transfer by diffusion can be heavily influenced by molecular clustering. Cluster theory in concentrated solutions can be approached using the Cussler model, which has been used to explain experimental mutual diffusion data in highly concentrated solutions. In this work, using the Cussler model and the critical point theory as a starting point, a new model for predictions of mutual diffusion coefficients in binary mixtures over the whole composition range was developed. The model is based on modifications of the concentration correlation function and explains well the experimental mutual diffusion data and their dependence on composition and activity coefficients. The model does not require any knowledge of intra-diffusion coefficients and can be used to predict mutual diffusion coefficients over the whole composition range.

Keywords: Diffusion; Cussler equation; Cluster theory; Concentrated solution; Thermodynamics

* Corresponding author. E-mail addresses: rahimpor@shirazu.ac.ir (M.R. Rahimpour). 


\section{Introduction}

2 Molecular diffusion in a single phase, or between two different phases, is a physical

3 phenomenon central to many important fields such as petroleum engineering, biotechnology,

4 catalysis and soil science [1]. For a proper design of many industrial unit operations and

5 modelling purposes, an accurate prediction and rationalization of mutual diffusion is often

6 needed [2]. Several methods can be used to measure mutual diffusion. D'Errico et al. [3]

7 used the Taylor dispersion method to measure mutual diffusion coefficients of glycerol/water

8 at $25{ }^{\circ} \mathrm{C}$. Other methods such as holographic interferometry, light scattering and diaphragm

9 cell methods have been used for prediction of mutual diffusion coefficients [4-6]. These methods are often complex to set-up, lengthy and in many cases suffer from accuracy and reproducibility issues. Models able to predict mutual diffusion coefficients can therefore offer a valid alternative.

Darken used the following expression for the prediction of mutual diffusion coefficients [7]:

$D_{12}=\left(x_{2} D_{1}^{*}+x_{1} D_{2}^{*}\right)\left[1+\frac{d \ln \gamma_{1}}{d \ln x_{1}}\right]$

14 In the model mentioned above, $D_{1}^{*}$ and $D_{2}^{*}$ are the intra-diffusion coefficient; $\gamma$ and $x$ are the activity coefficient and mole fraction, respectively. The term in the bracket is denoted as the thermodynamic correction factor. For ideal or near-ideal liquid systems, Darken's predictions are in good agreement with experimental data; however, for non-ideal liquid systems, the accuracy of the model becomes poor [8]. Different researchers have tried to modify the Darken equation to improve predictions [9-11].

D'Agostino et al. [2] modified the Darken equation and developed a model to predict diffusion coefficients in binary liquid systems using intra-diffusion coefficients at infinite dilution and viscosity data. In addition, D'Agostino and co-workers were also able to predict 
1 mutual diffusion coefficients in a non-ideal mixtures of hexane-nitrobenzene near the critical

2 point, using NMR diffusion data [12]. Their results showed improved prediction ability

3 relative to previous models and were in good agreement with the measured values of mutual

4 diffusion coefficients. Their method needs the knowledge of intra-diffusion experimental

5 data, either from NMR or radioactive tracer techniques, which are not always available. Other

6 approaches for prediction of mutual diffusion coefficients are reported in the literatures [13,

7 14].

8 One important aspect to consider is that of how diffusion in highly-concentrated liquid

9 systems can be physically explained and rationalized. In 1980, Cussler introduced the concept of cluster diffusion, which may occur in concentrated, non-ideal liquid solutions, where the movement of clusters or group of molecules, rather than single molecular species, dominates the diffusion process. In cluster diffusion, the local concentration as well as the local fluid velocity may undergo significant fluctuations [15]. Some researchers have predicted mutual diffusion coefficients using a scaling power for the thermodynamic correction factor, which accounts for these local concentration fluctuations, and developed models for non-ideal liquid systems [16-18]. For example, Moggridge [19] used the following equation for prediction of mutual diffusion in binary liquid mixtures containing one dimension species:

$D_{12}=\left(2 x_{2} D_{1}^{*}+x_{1} D_{2}^{*}\right)\left[1+\frac{d \ln \gamma_{1}}{d \ln x_{1}}\right]^{\alpha}$

where, $\alpha$ is a scaling power with a value of approximately 0.64 [20].

In this work, a model to analyze and predict mutual diffusion coefficients over the whole range of composition was developed, which is based on the modification of the concentration correlation function of cluster theory. The model explains well the experimental mutual diffusion data and their dependence on composition and activity coefficients. Accordingly, an optimization procedure was performed to determine the parameters of the developed model. The 
1 absolute relative error (ARD) was minimized in order to obtain the best fit of experimental

2 data. The interesting feature of proposed model is that it does not require any intra-diffusion

3 coefficient data.

4

5 2. Theory

6 The mutual diffusion coefficient in a liquid solution can usually be written according to the 7 following equation [15]:

$$
D=D_{0}\left(\frac{\partial \ln a_{1}}{\partial \ln x_{1}}\right)
$$

8 In the above equation, $D_{0}$ represents a mobility term and for a single spherical solute 9 molecule in a viscous continuum can be calculated from the Stokes-Einstein equation [15]

10 Mostly, equation (3) is incorrect for highly, non-ideal, concentrated solutions or solutions

11 near their consolute point, and this is attributed to the observation that in such solutions, 12 diffusion occurs through movement of clusters molecules alongside of single molecules [15].

13 The cluster diffusion approach can therefore be applied to study diffusion near consolute 14 point. In this region, concentration fluctuations become large [21]. In addition, near the consolute point, the mutual diffusion coefficient approaches zero [22]. As previously mentioned, in cluster diffusion, if fluctuations of velocity and concentration couple together,

17 the diffusion coefficient can be expressed as a time-integrated velocity and concentration correlation functions according to [23]:

$D=\frac{\langle G(r) * F(r)\rangle}{\langle G(r)\rangle}$

19 Where, $F(r)$ is the time-integrated velocity and $G(r)$ is the concentration function. In equation

(4) $G(r)$ and $F(r)$ are assumed to be independent of each other. 
1 Ferrell [24] obtained the time-integrated velocity correlation function according to the

2 following form:

$$
F_{i j}(\vec{r})=\frac{K T}{8 \pi \eta}\left(\frac{\delta_{i j}}{r}+\frac{r_{i} r_{j}}{r^{3}}\right)
$$

3 In the above equation, $T, \eta$ and $\delta_{i j}$ are the temperature, coefficient of viscosity and Kronecker 4 delta function, respectively. The trace of equation (5) is:

$$
F(r)=\frac{K T 1}{2 \pi \eta r}
$$

5 This equation describes random thermal movement of molecules (i.e., movement of 6 molecules because of their temperature).

7 Stanley [25] defined the $d$-dimensional Fourier transform of the concentration function 8 according to:

$$
G(r)=\int S(q) e^{-i q \cdot r} d q
$$

9 where $S(q)$ is the structure function shown in the equation below [25]:

$$
\frac{1}{n} S(q)=1+n \hat{\Gamma}(q)=\frac{1}{1-n \hat{C}(q)}
$$

10 In the above equation, $\hat{C}(q)$ is Fourier transform of $C(r)$, which is called direct correlation

11 function. Besides, $\hat{\Gamma}(q)$ is the Fourier transform of $\Gamma(r)$, where $\Gamma(r)$ is a dimensionless 12 parameter, which describes the effect of correlation between different particles in the 13 concentration function. The Taylor expansion of $\hat{C}(q)$ is shown in equation (9):

$$
\hat{C}(q)=\hat{C}(0)+\sum_{i=0}^{\infty} \hat{C}_{i}(n, T) q^{i}
$$

14 Hence, by combining equations (8) and (9), the following equation can be obtained: 
$\frac{n}{S(q)}=1-n \hat{C}(q)=1-n\left\{\hat{C}(0)+\hat{C}_{2}(n, T) q^{2}+\Theta\left(q^{4}\right)\right\}$

Defining:

$R^{2}=-n \hat{C}_{2}(n, T)$

1 and omitting all the terms of four order of $q$ and higher, Stanley obtained the concentration

2 function at consolute temperature for liquid systems [25]:

$G(r)=\frac{R^{2}}{r} \exp \left(-\frac{r}{\xi}\right)$

3 In the above equation $\xi \cong k^{-1}$, which is the characteristic size for the concentration

4 fluctuation [15]. $R^{2}$ is a length on order of molecular size and $k$ is called inverse correlation 5 length.

6 Equation (12) was obtained by omitting some parameters of high order. Since the term of $\Theta($

$7 \quad q^{4}$ ) is omitted in equation (10), we modified the concentration function in equation (12) with 8 the following equation:

$G(r)=\frac{R^{2}}{r^{n}} \exp \left(-\frac{r}{\xi}\right)$

9 In the above equation, $n$ is an adjustable parameter, which accounts for the omitted terms in

10 equation 10. Hence, by combining equations (4), (6) and (13), the modified diffusion

11 coefficient can be obtained according to:

$D=\frac{4 \pi \int_{0}^{\infty} \frac{a}{r^{n}} \exp \left(-\frac{r}{\xi}\right) * \frac{K T 1}{2 \pi \eta r} r^{2} d r}{4 \pi \int_{0}^{\infty} \frac{a}{r^{n}} \exp \left(-\frac{r}{\xi}\right) * r^{2} d r}=\frac{K T}{2 \pi \eta \xi(2-n)}$ 
1 Equation (14) can be identified as a modified cluster diffusion coefficient, where $\xi(2-n)$

2 and $K$ are the corrected length and Boltzmann constant, respectively. In the Stokes-Einstein

3 equation $6 \pi$ displaces $2 \pi$, which originates from the velocity correlation for spherical solute

4 molecules. The main difference between the Stokes-Einstein equation:

$D=\frac{K T}{6 \pi \eta r}$

5 and the modified diffusion coefficient equation, equation (14), is in terms of the characteristic

6 size, which is in a modified form, $\xi$, substituted instead of the radius $r$.

7 In order to use equation (14), $\xi$ must be estimated. The relation between $\xi$ and chemical

8 potential is given as follows [26]. It should be mentioned that near the consolute point the

9 common models for the chemical potential are not accurate.

$$
\begin{aligned}
& \frac{1 \partial \mu_{1}}{k T \partial x_{1}}=\frac{1}{x_{1}}-\frac{m x_{2}\left(g_{11}+g_{22}-2 g_{12}\right)}{1+m x_{1} x_{2}\left(g_{11}+g_{22}-2 g_{12}\right)} \\
& g_{i j}=4 \pi \int_{0}^{\infty} G_{i j} r^{2} d r
\end{aligned}
$$

10 where, $m$ is the number of molecules per unit volume and $G_{i j}$ is the radial concentration

11 distribution function, which has the Ornstein-Zernike form [25]. By combining equations

12 (13) and (17), $g_{i j}$ can be obtained as:

$g_{i j}=4 \pi \int_{0}^{\infty} a_{i j} r^{2-n} \exp \left(-\frac{r}{\xi_{i j}}\right) d r=4 \pi a_{i j} \xi^{3-n} \Gamma(3-n)$

13 In the above equation, $a_{i j}$ is a characteristic of molecular size. To simplify equation (18), we

14 assume that:

$$
\begin{aligned}
& 4 \pi a \Gamma(3-n) \xi^{3-n} \simeq \\
& 4 \pi a_{11} \xi^{3-n} \Gamma(3-n)+4 \pi a_{22} \xi^{3-n} \Gamma(3-n)-8 \pi a_{12} \xi_{12}^{3-n} \Gamma(3-n)
\end{aligned}
$$


1 By combining equations (16) and (19), $\xi$ was found according to:

$\xi=\left(\frac{1}{4 \pi a m \Gamma(3-n)} * \frac{1}{x_{1} x_{2}}\left(\frac{k T \partial x_{1}}{x_{1} \partial \mu_{1}}-1\right)\right)^{\frac{1}{3-n}}=\left(Y * \frac{1}{x_{1} x_{2}}\left(\frac{k T \partial x_{1}}{x_{1} \partial \mu_{1}}-1\right)\right)^{\frac{1}{3-n}}$

with

$Y=\frac{1}{4 \pi a m \Gamma(3-n)}$

2 The correlation length that is predicted by equation (20) has no physical meaning in an ideal

3 solution, because it predicts a correlation length of zero. However, the correlation length

4 cannot be smaller than the average diameter of the molecules and in an ideal solution is

5 assumed to be $\xi=\beta r_{0}$, where $\beta$ has generally a specific value for each individual system.

6 Therefore, equation (20) was modified empirically and the diffusion coefficient can be

7 written as:

$D=\frac{K T}{2 \pi \eta(2-n) \beta r_{0}}\left(\frac{1}{1+Y_{1} * \frac{1}{x_{1} x_{2}}\left(\frac{k T \partial x_{1}}{x_{1} \partial \mu_{1}}-1\right)}\right)^{\frac{1}{3-n}}$

8 where $r_{0}$ is the radius of the solute molecules. In this way, the correlation length inevitably

9 reduces to the Stokes-Einstein equation for dilute solutions, without affecting the results near

10 the critical point. Indeed, the second term in equation (22), $\left(\frac{1}{1+Y_{1} * \frac{1}{x_{1} x_{2}}\left(\frac{k T \partial x_{1}}{x_{1} \partial \mu_{1}}-1\right)}\right)^{\frac{1}{3-n}}$, is the

11 correction factor.

12 For a non-ideal solution, the chemical potential is defined as:

$\mu_{1}=k T \ln \left(\gamma_{1} x_{1}\right)+\mu_{1}^{0}$

13 For calculation of diffusion coefficients, a model for activity coefficients $\left(\gamma_{i}\right)$ is needed.

14 Combining equations (22) and (23) gives us: 


$$
D=\frac{K T}{2 \pi \eta(2-n) \beta r_{0}}\left(\frac{1}{1+Y_{1} * \frac{1}{x_{1} x_{2}}\left(\frac{1}{1+\frac{d \ln \gamma_{1}}{d \ln x_{1}}}-1\right)}\right)^{\frac{1}{3-n}}
$$

1 There are three adjustable parameters in equation (24) $\left(\beta, n, Y_{1}\right)$. These three parameters

2 were determined by optimization.

3 The correction factor in equation (24) was calculated from vapor-liquid equilibrium (VLE)

4 data. For the calculation of activity coefficients, equation (25) was used and $P, x, y$ data were

5 collected from the literature for each binary system [27].

$$
\gamma_{i}=\frac{y_{i} P}{x_{i} p_{i}^{s a t}}
$$

6 In equation (23), for the calculation of $\frac{d \ln \gamma_{1}}{d \ln x_{1}}$, a fourth order Runge-Kutta algorithm with step

7 of 0.005 was used. Vapor mole fraction could be calculated from the following equation:

$$
d y_{i}=\frac{y_{i}\left(1-y_{i}\right)}{\left(y_{i}-x_{i}\right) P} d P
$$

8 Activity coefficients were determined as a function of composition. In this regard, VLE data

9 were fitted using second to six degree polynomials and if the results were not satisfactory, equation (27) would be used to describe the total vapor pressure:

$P=P_{j}^{s a t}+\left\{\left[A+B x_{i}\right]\left[1-\exp \left(C x_{i}\right)\right]\right\}$

11 In the above equation, $\mathrm{A}, \mathrm{B}$ and $\mathrm{C}$ are adjustable parameters.

12 It should be noted that equilibrium data $(x, y$ and $P)$ and diffusion coefficients were reported

13 at two slightly different temperatures in the literature, thus the calculation of $\frac{d \ln \gamma_{1}}{d \ln x_{1}}$ by

14 extrapolation was affected by error. If both VLE experimental data and diffusion coefficient

15 are exactly at same temperature, the accuracy of the calculations can be improved. The 
1 viscosity data, which is used in the present contribution, are collected from available open

2 literature data $[2,28-32]$

3 To show the capability of the proposed model, this is compared with the results of Cussler $4 \quad[15]:$

$$
D=D_{0}\left[\frac{1}{1+\frac{K_{c}}{x_{1} x_{2}}\left[\frac{k T \partial x_{1}}{x_{1} \partial \mu_{1}}-1\right]}\right]^{\frac{1}{2}}
$$

\section{2.1. Parameter Evaluation}

6 The parameters $\left(\beta, n, Y_{1}\right)$ in equation (24) are optimized to the experimental mutual diffusion

7 data with an objective function defined in equation (29). The absolute relative error (ARD)

8 was minimized in order to have the best fit of experimental data. In this regard, simplex 9 search method [33] was applied as an appropriate non-linear regression strategy. All the 10 collected experimental diffusivity data were in the temperature range of $20-25^{\circ} \mathrm{C}$.

$A R D \%=\frac{100}{N D P} \sum_{i=1}^{N D P}\left|\frac{D^{\text {exp. }}-D^{\text {cal. }}}{D^{\text {exp. }}}\right|$

\section{Results and discussion}

12 Experimental mutual diffusion coefficient data for alcohol-carbon tetrachloride at $20{ }^{\circ} \mathrm{C}$ were

13 collected from the work of Sanchez and Clifton [4] and those of methanol-benzene and 14 alcohol-carbon disulfide for temperature range of $20{ }^{\circ} \mathrm{C}$ to $80{ }^{\circ} \mathrm{C}$ were collected from the work 15 of McKeigue and Gulari [5]. In addition, experimental equilibrium data of alcohol-carbon 16 tetrachloride at two temperature of $35^{\circ} \mathrm{C}$ and those of alcohol-carbon disulfide at $30{ }^{\circ} \mathrm{C}$ were 
1 reported in the works of Paraskevopoulos and Missen [30] and McKeigue and Gulari [32],

2 respectively.

3 The three adjustable parameters in equation (24) were obtained for seven different systems.

4 The local and global parameters are tabulated in Table 1. Furthermore, a comparison between

5 this model and that of Cussler was also carried out. Accordingly, the Cussler model includes

6 a $K_{c}$ parameter, which is a fitting parameter that varies with different binary systems.

Table 1: The best-fit parameters of the proposed model and Cussler model

\begin{tabular}{lcccc}
\hline & \multicolumn{3}{c}{ New Model } & Cussler Model \\
\cline { 2 - 5 } System & $\boldsymbol{Y}_{\boldsymbol{1}}$ & $\boldsymbol{n}$ & $\boldsymbol{\beta}$ & $\boldsymbol{K}_{\boldsymbol{c}}$ \\
\hline 1-Butanol +Carbon tetrachloride & 7.853 & 0.789 & 0.182 & 0.003 \\
1-Propanol+carbon tetrachloride & 3.658 & -0.141 & 0.196 & -0.002 \\
Ethanol + Carbon tetrachloride & 12.725 & -0.444 & 0.155 & 0.003 \\
Methanol + Carbon tetrachloride & 1.790 & 0.801 & 0.294 & 0.016 \\
1-Butanol + Carbon disulfide & 24.89 & 0.762 & 0.117 & 0.017 \\
1-Pentanol + Carbon disulfide & 53.875 & 0.744 & 0.109 & 0.028 \\
Methanol +Benzene & 11.009 & 1.014 & 0.127 & 0.01 \\
Global & 2.245 & 0.515 & 0.286 & - \\
\hline
\end{tabular}

8

9 It is interesting to note that the optimized $n$ global value yield an exponential of the thermodynamic correction factor, $1 /(3-n)$ of approximately 0.402 , which is close to the 0.5 value given by Cussler for the power of the correction factor.

The theoretical model was applied to seven different systems and the results explain well the mutual diffusion profiles. It has to be pointed out that because this model is based on cluster theory, in most cases it predicts experimental data at high concentration better than those at low concentrations. Such observation is in line with the theoretical basis of the model (i.e., cluster theory), which is applied in the high concentration range.

We now assess the use of the model in equation (24) to evaluate diffusion coefficient data using the global parameters; the obtained results are compared with those obtained with local parameters in Figures 1-3 and Table 2. The main aim is to verify whether we can obtain 
1 reasonable predictions using a cluster theory model with parameters that are independent of

2 the system, hence can be of more general validity and can potentially be applied to predict

3 data for systems in which experimental values of mutual diffusion are not available. This is a

4 main difference with the Cussler model, which requires a single parameter that, however,

5 varies with the binary system.

6

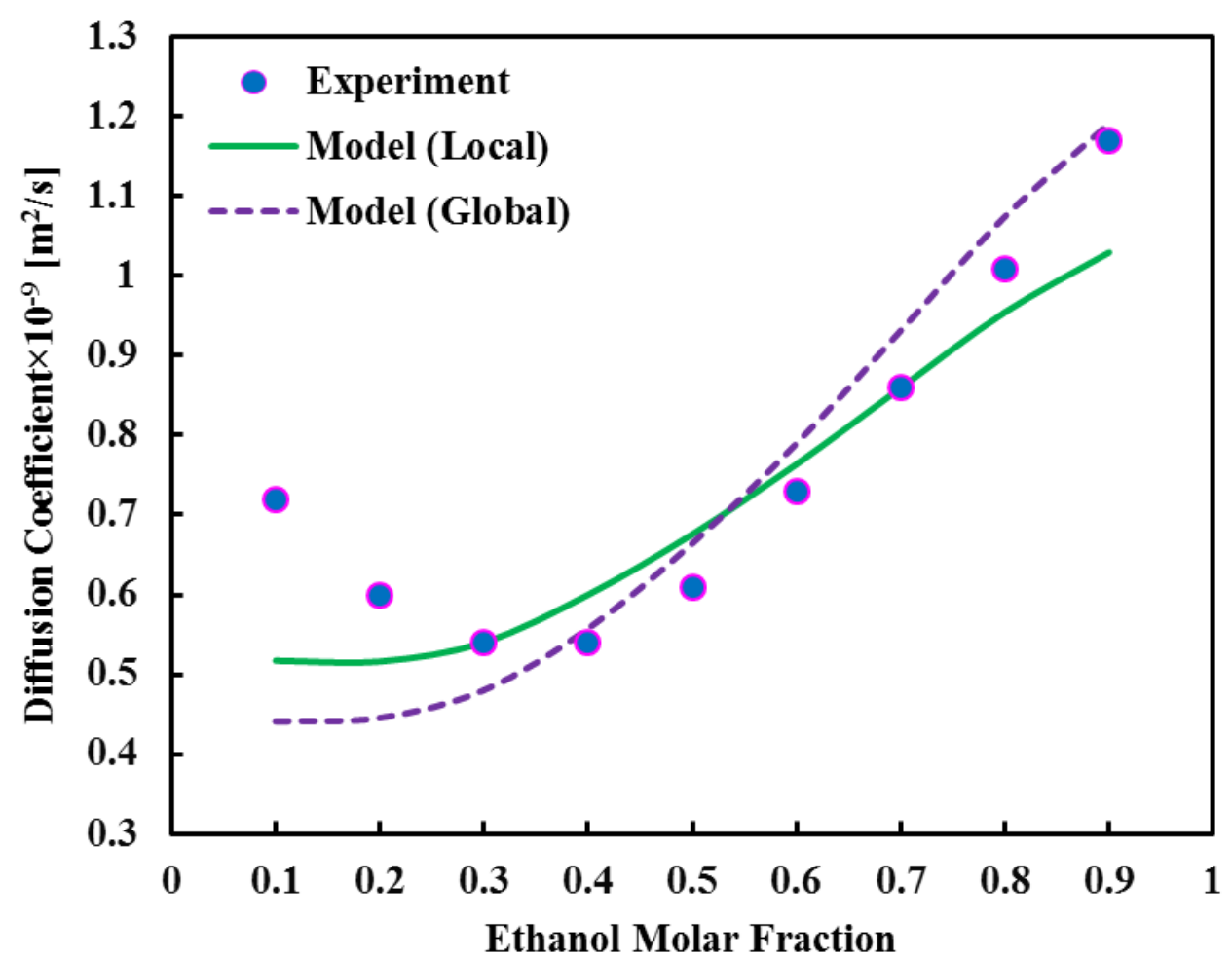

7

8 Fig 1. Diffusion coefficients for ethanol + carbon tetrachloride system with both local and global parameters. 


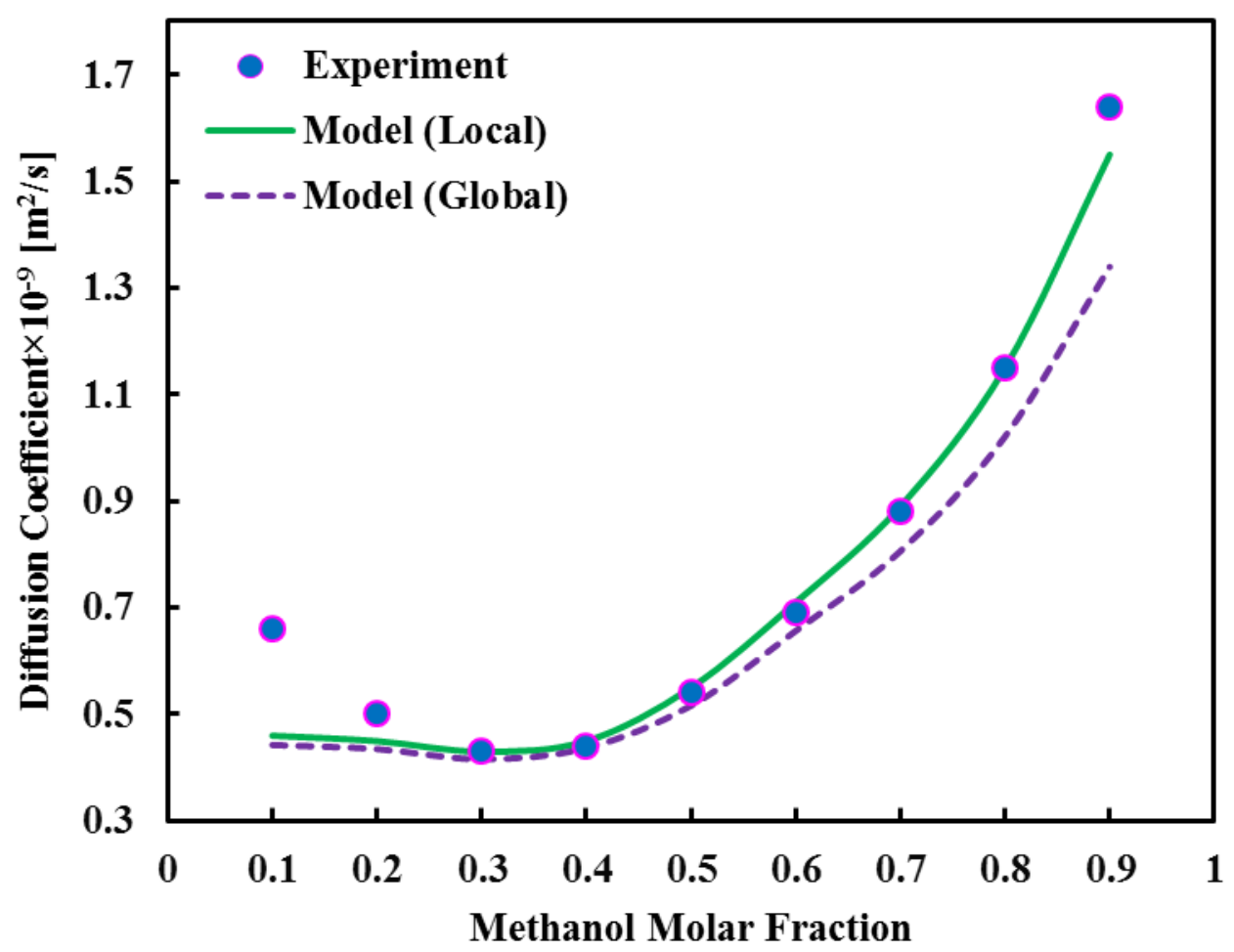

1

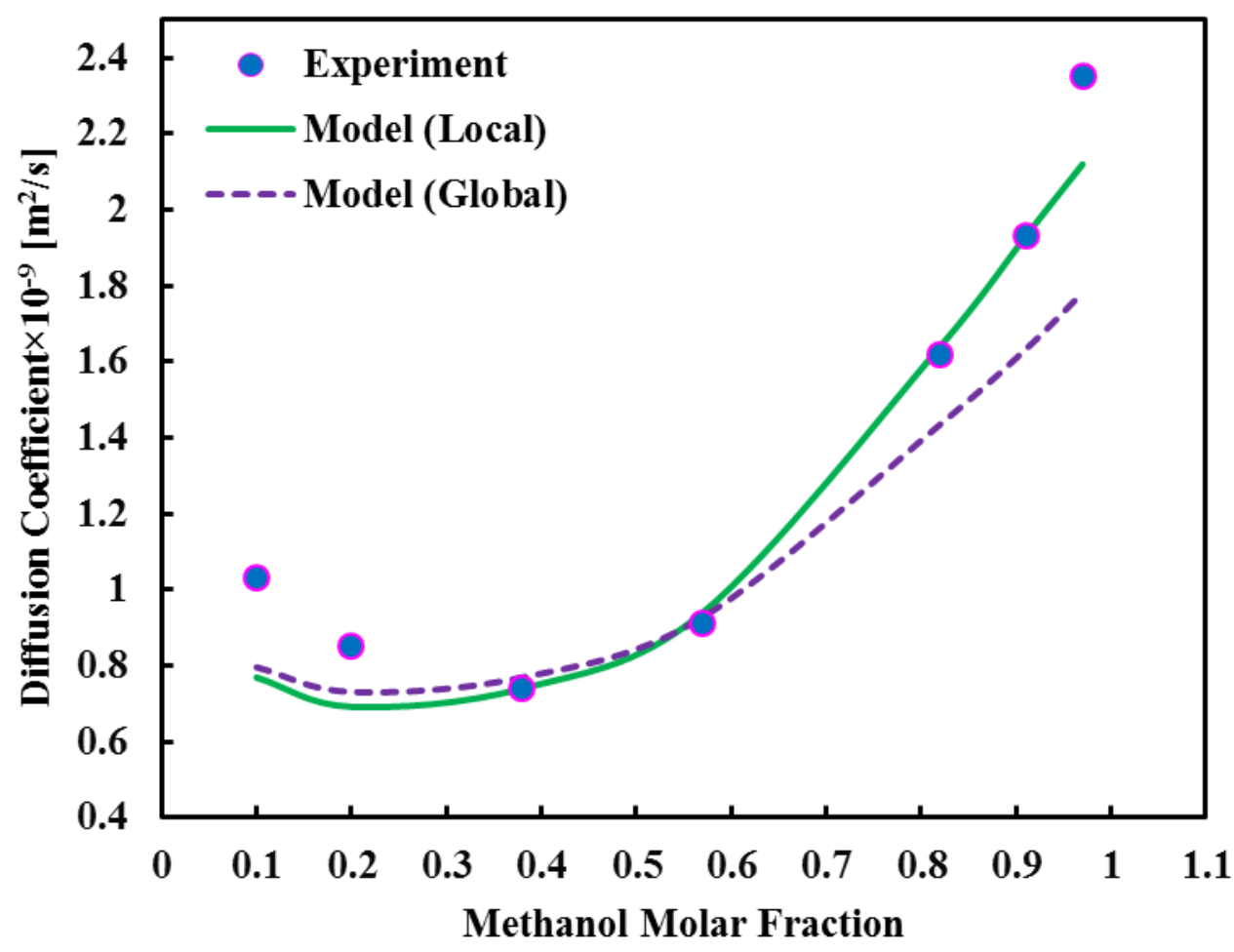

4

$5 \quad$ Fig 3. Diffusion coefficients for methanol + benzene with both local and global parameters. 
1 The ARD \% results for local and global parameters are presented in Table 2. The focus is in

2 assessing how the model performs when global parameters are used. It can be seen that in

3 most cases our model with global parameters gives lower errors in predictions compared to

4 Cussler. A very high error is observed for the 1-Pentanol+Carbon disulfide system, for which

5 our model does not perform well and the error is higher than. However, the measured ARD \%

6 for this system is significantly higher compared to the other systems also when using the

7 Cussler model, which could be due to other reasons such as inaccuracy of thermodynamic

8 data and use of diffusion and thermodynamic data at slightly different temperatures, as

9 previously mentioned. However, in general, it can be seen that the overall ARD \% of our

10 model with global parameters is improved.

Table 2 Comparison of error (ARD) of new model with those of Cussler.

\begin{tabular}{lcccc}
\hline ARD\% & $\begin{array}{c}\text { This work (Local } \\
\text { Parameters) }\end{array}$ & $\begin{array}{c}\text { This work } \\
\text { (Global } \\
\text { Parameters) }\end{array}$ & $\begin{array}{c}\text { Cussler } \\
\text { Model }\end{array}$ & $\begin{array}{c}\text { P-x-y experimental } \\
\text { data }\end{array}$ \\
\hline $\begin{array}{l}\text { 1-Butanol +Carbon } \\
\text { tetrachloride }\end{array}$ & 4.2 & 6.29 & 29.05 & 5.06 \\
$\begin{array}{l}\text { 1-Propanol+carbon } \\
\text { tetrachloride }\end{array}$ & 6.46 & 13.02 & 25.30 & 7.20 \\
$\begin{array}{l}\text { Ethanol + Carbon } \\
\text { tetrachloride }\end{array}$ & 9.39 & 12.53 & 21.80 & 8.46 \\
$\begin{array}{l}\text { Methanol + Carbon } \\
\text { tetrachloride }\end{array}$ & 5.97 & 10.79 & 19.54 & 5.90 \\
$\begin{array}{l}\text { 1-Butanol + Carbon } \\
\text { disulfide }\end{array}$ & 10.85 & 24.28 & 23.03 & 9.26 \\
$\begin{array}{l}\text { 1-Pentanol + Carbon } \\
\text { disulfide }\end{array}$ & 15.5 & 52.76 & 22.97 & 8.20 \\
$\begin{array}{l}\text { Methanol +Benzene } \\
\text { Overall }\end{array}$ & 8.05 & 13.38 & 24.83 & 4.36 \\
\hline a & 8.63 & 17.08 & 23.78 & - \\
\hline
\end{tabular}

15 Furthermore, to show the prediction capability of the model, mutual diffusion coefficient were predicted by using local and global parameters. The results are presented in figures 4

17 and 5. The results show that calculated mutual diffusion coefficients are in good agreement 
1 with the experimental ones and that in most cases the use of the model with the optimized

2 global parameters gives reasonably good results. This show that the model can be of a more 3 general validity.

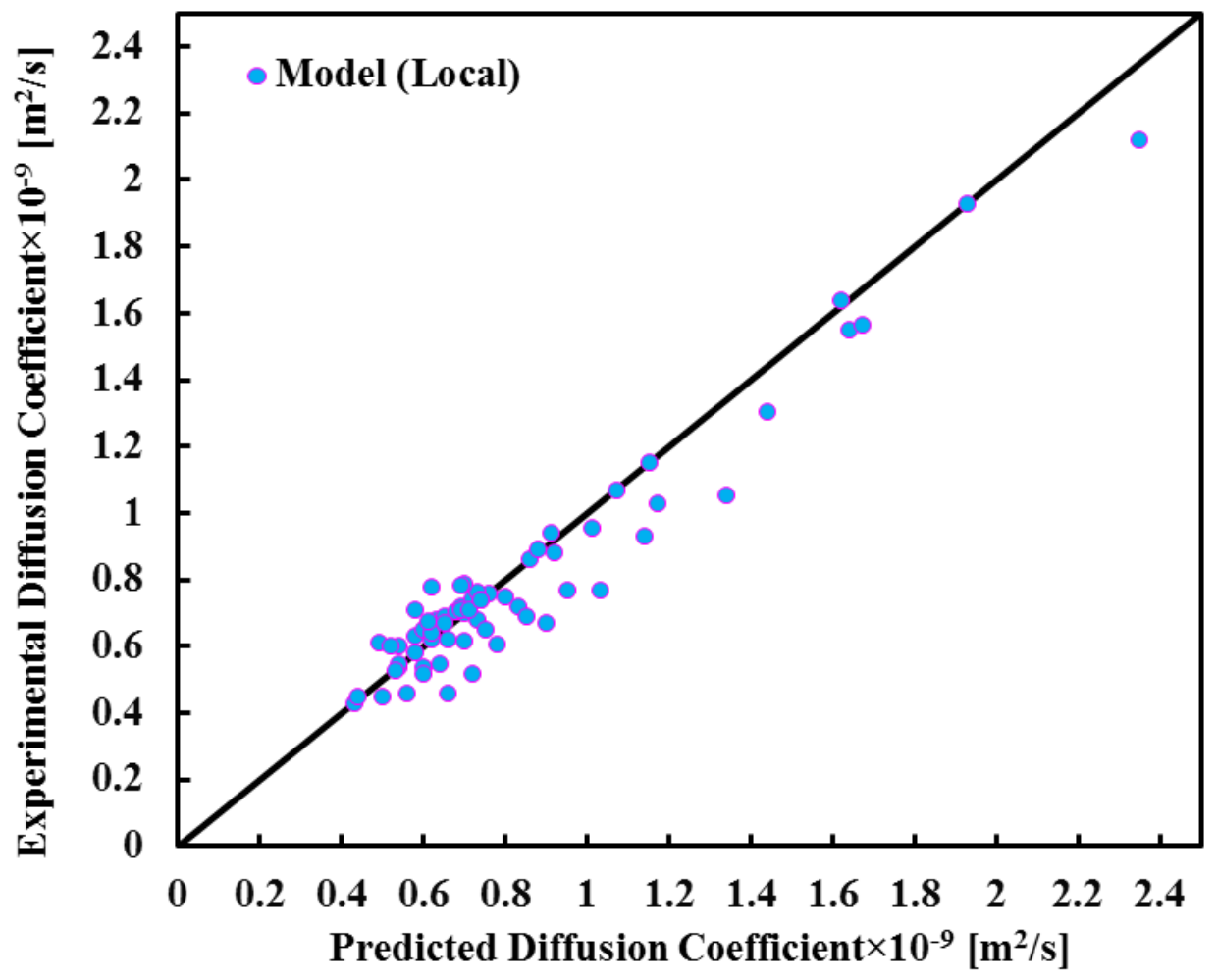

4

Fig 4. Prediction of diffusion coefficient using local parameters 


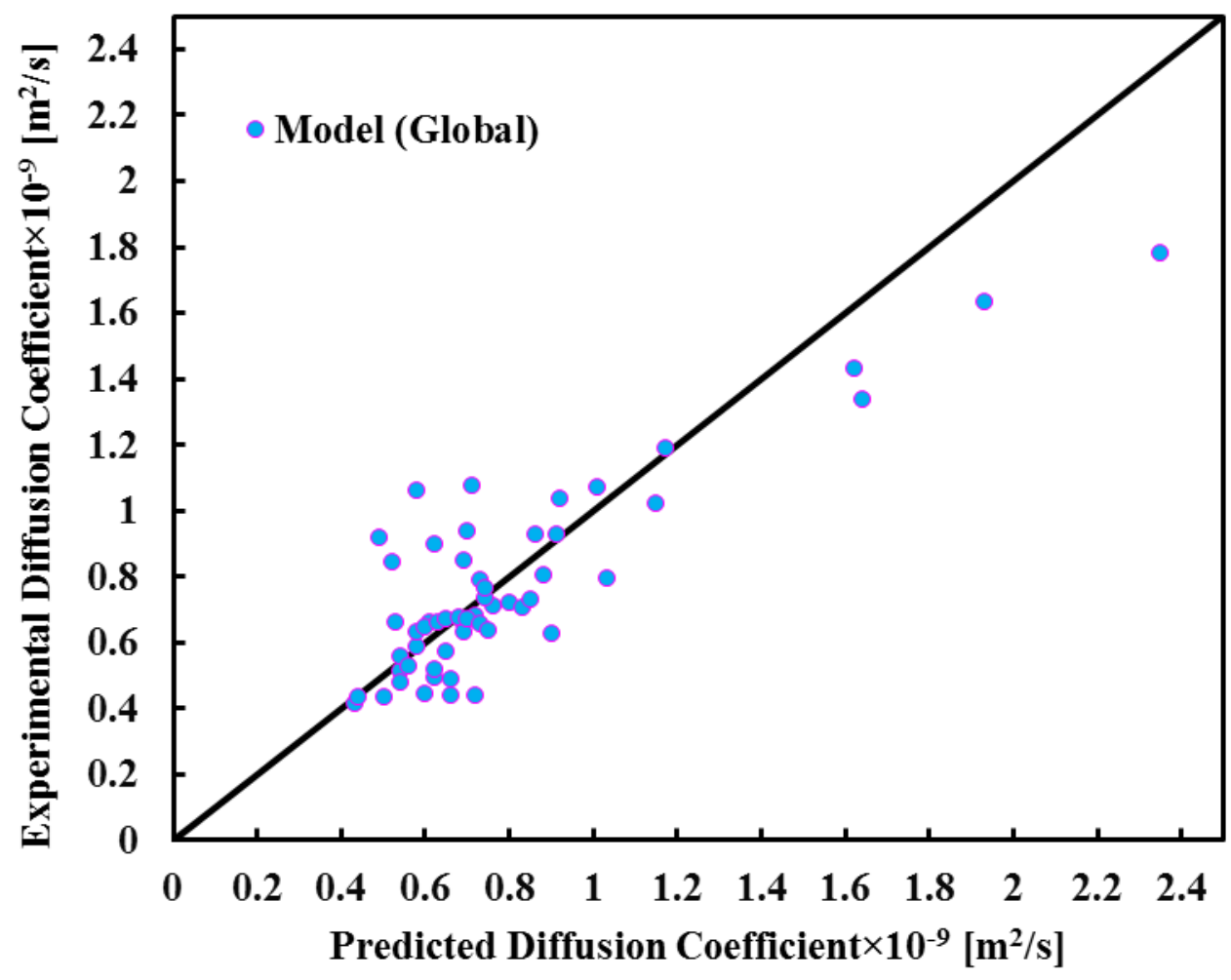

1

Fig 5. Prediction of diffusion coefficient using local parameters

\section{Conclusion}

5 In the present contribution, a model based on cluster theory of Cussler with the modifications

6 of the concentration correlation function was proposed for the description of mutual diffusion

7 coefficients in non-ideal, concentrated binary mixtures. Local and global parameters of the

8 model were evaluated by optimization. The aim was to see if a cluster theory model with

9 global parameters can be used to model and predict mutual diffusion data. Generally, the 10 model describes well the mutual diffusion data reported in the literature. The model does not

11 need any knowledge on intra-diffusion coefficients and can be used to estimate mutual diffusion coefficients as a function of composition when such data are not available. 
1. Jamialahmadi, M., M. Emadi, and H. Müller-Steinhagen, Diffusion coefficients of methane in liquid hydrocarbons at high pressure and temperature. Journal of Petroleum Science and Engineering, 2006. 53(1): p. 47-60.

2. Zhu, Q., et al., Prediction of mutual diffusion coefficients in binary liquid systems with one self-associating component from viscosity data and intra-diffusion coefficients at infinite dilution. Chemical Engineering Science, 2016. 147: p. 118-127.

3. D'Errico, G., et al., Diffusion coefficients for the binary system glycerol + water at 25 C. A velocity correlation study. Journal of Chemical \& Engineering Data, 2004. 49(6): p. 16651670 .

4. Sanchez, V. and M. Clifton, Mutual diffusion coefficients in binary mixtures of carbon tetrachloride and alcohols at 20. degree. C. Journal of Chemical and Engineering Data, 1978. 23(3): p. 209-212.

5. McKeigue, K. and E. Gulari, Light-scattering measurements of diffusion in binary solutions containing an associating component. The Journal of Physical Chemistry, 1984. 88(16): p. 3472-3479.

6. Cain, J., J. Clunie, and J. Baird, Diaphragm cell determination of the interdiffusion coefficient for succinonitrile + water. International journal of thermophysics, 1995. 16(5): p. 1225-1234.

7. Darken, L., Diffusion, Mobility and Their Interrelation through Free Energy in Binary Metallic Systems. Metallurgical and materials transactions. B, Process metallurgy and materials processing science, 2010. 41(2): p. 277-294.

8. Pertler, M., E. Blass, and G.W. Stevens, Fickian diffusion in binary mixtures that form two liquid phases. AIChE journal, 1996. 42(4): p. 910-920.

9. Zhou, M., et al., Local composition based Maxwell-Stefan diffusivity model for binary liquid systems. Industrial \& Engineering Chemistry Research, 2013. 52(31): p. 10845-10852.

10. Moggridge, G., Prediction of the mutual diffusivity in binary non-ideal liquid mixtures from the tracer diffusion coefficients. Chemical engineering science, 2012. 71: p. 226-238.

11. Hsu, Y.-D., M. Tang, and Y.-P. Chen, A group contribution correlation of the mutual diffusion coefficients of binary liquid mixtures. Fluid phase equilibria, 2000. 173(1): p. 1-21.

12. D'Agostino, C., et al., Prediction of binary diffusion coefficients in non-ideal mixtures from NMR data: hexane-nitrobenzene near its consolute point. Chemical engineering science, 2011. 66(17): p. 3898-3906.

13. Tharanivasan, A.K., C. Yang, and Y. Gu, Comparison of three different interface mass transfer models used in the experimental measurement of solvent diffusivity in heavy oil. Journal of Petroleum Science and Engineering, 2004. 44(3): p. 269-282.

14. Sekerci-Cetin, M., et al., Numerical study of the dissolution of carbon dioxide in an ionic liquid. Chemical Engineering Science, 2016. 147: p. 173-179.

15. Cussler, E., Cluster diffusion in liquids. AIChE Journal, 1980. 26(1): p. 43-51.

16. D'Agostino, C., et al., Prediction of the mutual diffusivity in acetone-chloroform liquid mixtures from the tracer diffusion coefficients. Chemical Engineering Science, 2013. 95: p. 43-47.

17. D'Agostino, C., et al., Hydrogen bonding network disruption in mesoporous catalyst supports probed by PFG-NMR diffusometry and NMR relaxometry. The Journal of Physical Chemistry C, 2012. 116(16): p. 8975-8982.

18. D'Agostino, C., et al., Prediction of mutual diffusion coefficients in non-ideal mixtures from pulsed field gradient NMR data: Triethylamine-water near its consolute point. Chemical engineering science, 2012. 74: p. 105-113.

19. Moggridge, G., Prediction of the mutual diffusivity in binary liquid mixtures containing one dimerising species, from the tracer diffusion coefficients. Chemical engineering science, 2012. 76: p. 199-205.

20. De, S., Y. Shapir, and E. Chimowitz, Scaling of self and Fickian diffusion coefficients in the critical region. Chemical engineering science, 2001. 56(17): p. 5003-5010. 
21. Swift, J., Transport coefficients near the consolute temperature of a binary liquid mixture. Physical Review, 1968. 173(1): p. 257.

22. Haase, R. and M. Siry, Diffusion im kritischen Entmischungsgebiet binärer flüssiger Systeme. Zeitschrift für Physikalische Chemie, 1968. 57(1_2): p. 56-73.

23. Ferrell, R.A., Decoupled-Mode Dynamical Scaling Theory of Phase Transitions. Dynamical Aspects of Critical Phenomena, JI Budnick and MP Kawatra Gordon and Breach, New York, 1972.

24. Ferrell, R.A., Decoupled-mode dynamical scaling theory of the binary-liquid phase transition. Physical Review Letters, 1970. 24(21): p. 1169.

25. Stanley, H., Introduction to Phase Transitions and Critical PhenomenaOxford Univ. Press. 1971, Oxford.

26. Kirkwood, J.G. and F.P. Buff, The statistical mechanical theory of solutions. I. The Journal of chemical physics, 1951. 19(6): p. 774-777.

27. Shiflett, M.B. and A. Yokozeki, Solubility and diffusivity of hydrofluorocarbons in room-temperature ionic liquids. AIChE journal, 2006. 52(3): p. 1205-1219.

28. González, B., Á. Domínguez, and J. Tojo, Dynamic viscosities of the binary systems cyclohexane and cyclopentane with acetone, butanone, or 2-pentanone at three temperatures $T=(293.15,298.15$, and 303.15) $K$. Journal of Chemical \& Engineering Data, 2005. 50(4): p. 1462-1469.

29. Nikam, P.S. and A.B. Nikumbh, Ionic viscosity b-coefficients of tetraalkyl ammonium chlorides in (0 to 100) mass water+ methanol at $298.15 \mathrm{~K}$. Journal of Chemical \& Engineering Data, 2002. 47(3): p. 400-404.

30. Paraskevopoulos, G. and R. Missen, Thermodynamic properties of solutions of alcohols and carbon tetrachloride. Part 1.-Free energies and volumes of mixing. Transactions of the Faraday Society, 1962. 58: p. 869-878.

31. Li, J., H. Liu, and Y. Hu, A mutual-diffusion-coefficient model based on local composition. Fluid phase equilibria, 2001. 187: p. 193-208.

32. McKeigue, K. and E. Gulari, Vapor-liquid equilibria for binary mixtures of 1-alcohols in carbon disulfide. Journal of Chemical and Engineering Data, 1986. 31(3): p. 278-283.

33. Lagarias, J.C., et al., Convergence properties of the Nelder--Mead simplex method in low dimensions. SIAM Journal on optimization, 1998. 9(1): p. 112-147. 
- In this research cluster theory was modified

- An improved model was proposed to predict mutual diffusion coefficient

- Seven binary systems are used to validate the credentials of the model

- This model showed better consistency in comparison with another model 